Louisiana State University

LSU Digital Commons

Faculty Publications

Department of Biological Sciences

$12-1-2008$

\title{
Host-pathogen interactions, insect outbreaks, and natural selection for disease Resistance
}

\author{
Bret D. Elderd \\ The University of Chicago \\ Jonathan Dushoff \\ McMaster University \\ Greg Dwyer \\ The University of Chicago
}

Follow this and additional works at: https://digitalcommons.Isu.edu/biosci_pubs

\section{Recommended Citation}

Elderd, B., Dushoff, J., \& Dwyer, G. (2008). Host-pathogen interactions, insect outbreaks, and natural selection for disease Resistance. American Naturalist, 172 (6), 829-842. https://doi.org/10.1086/592403

This Article is brought to you for free and open access by the Department of Biological Sciences at LSU Digital Commons. It has been accepted for inclusion in Faculty Publications by an authorized administrator of LSU Digital Commons. For more information, please contact ir@lsu.edu. 


\section{Host-Pathogen Interactions, Insect Outbreaks, and Natural Selection for Disease Resistance}

Bret D. Elderd, ${ }^{1, *}$ Jonathan Dushoff, ${ }^{2}$ and Greg Dwyer ${ }^{1, \dagger}$

1. Department of Ecology and Evolution, University of Chicago, Chicago, Illinois 60637;

2. Department of Biology, McMaster University, West Hamilton, Ontario L8S 4K1, Canada

Submitted August 7, 2007; Accepted July 16, 2008;

Electronically published October 31, 2008

Online enhancement: appendix.

ABstract: The theory of insect population dynamics has shown that heterogeneity in natural-enemy attack rates is strongly stabilizing. We tested the usefulness of this theory for outbreaking insects, many of which are attacked by infectious pathogens. We measured heterogeneity among gypsy moth larvae in their risk of infection with a nucleopolyhedrovirus, which is effectively heterogeneity in the pathogen's attack rate. Our data show that heterogeneity in infection risk in this insect is so high that it leads to a stable equilibrium in the models, which is inconsistent with the outbreaks seen in North American gypsy moth populations. Our data further suggest that infection risk declines after epidemics, in turn suggesting that the model assumption of constant infection risk is incorrect. We therefore constructed an alternative model in which natural selection drives fluctuations in infection risk, leading to reductions after epidemics because of selection for resistance and increases after epidemics because of a cost of resistance. This model shows cycles even for high heterogeneity, and experiments confirm that infection risk is indeed heritable. The model is very general, and so we argue that natural selection for disease resistance may play a role in many insect outbreaks.

Keywords: gypsy moth (Lymantria dispar), host-pathogen interactions, evolution of resistance, nucleopolyhedrovirus (NPV), complex dynamics.

* Present address: Department of Biological Sciences, Louisiana State University, Baton Rouge, Louisiana 70803.

† Corresponding author; e-mail: gdwyer@uchicago.edu.

Am. Nat. 2008. Vol. 172, pp. 829-842. (c) 2008 by The University of Chicago. 0003-0147/2008/17206-42794\$15.00. All rights reserved.

DOI: $10.1086 / 592403$
A basic result from the theory of insect population dynamics is that heterogeneity in natural-enemy attack rates is strongly stabilizing. This result is perhaps best known as the $\mathrm{CV}^{2}>1$ rule (Hassell et al. 1991), which states that a stable equilibrium is guaranteed if the squared coefficient of variation $(\mathrm{CV})$ of attack rates is $>1$. The $\mathrm{CV}^{2}>1$ rule applies to both host-parasitoid and host-pathogen models, but the two types of models differ in an important way. In host-parasitoid models, $\mathrm{CV}^{2}<1$ results in unstable cycles, but in host-pathogen models, there is a range of $\mathrm{CV}^{2}$ values $<1$ for which stable cycles occur (Dwyer et al. 2000). This is important because stable cycles provide the best explanation for the periodic outbreaks often observed in insects that are attacked by pathogens (Moreau and Lucarotti 2007).

Here we use experiments to show that heterogeneity in the attack rate of an insect pathogen is too high to allow stable cycles in existing models, and we propose an alternative general model to explain insect outbreaks. The pathogen that we use is a nucleopolyhedrovirus (NPV) of the gypsy moth Lymantria dispar, which undergoes outbreaks in North America (Elkinton and Liebhold 1990). Insect NPVs are transmitted when host larvae consume foliage contaminated with infectious particles, known as occlusion bodies, that allow survival outside of the host (Cory and Myers 2003). Because only larvae can become infected, there can be only one epidemic per generation. The epidemic is begun when hatching larvae are infected with occlusion bodies that have survived in the environment since the previous epidemic (Murray and Elkinton 1989, 1990). The NPV transmission cycle is thus simple enough that we can model it using the general epidemic model of Kermack and McKendrick (1927; see also Dwyer and Elkinton 1993),

$$
\begin{aligned}
& \frac{d S}{d t}=-\nu S P, \\
& \frac{d P}{d t}=\nu P(t-\tau) S(t-\tau)-\mu P,
\end{aligned}
$$

where $S$ and $P$ are the densities of uninfected hosts and 
infectious cadavers, respectively, so that transmission occurs with rate parameter $\nu$. After $\tau$ time units, infected larvae die and become infectious cadavers at time $t$, releasing occlusion bodies onto the foliage to complete the cycle of transmission (Cory and Myers 2003). Occlusion bodies are rendered inactive by sunlight and other environmental factors at a rate $\mu$.

A simple way to allow for heterogeneity is to assume that attack rates follow a probability distribution (Hassell et al. 1991). In epidemic models, the transmission parameter $\nu$ is effectively the natural-enemy attack rate, and so we allow for a probability distribution of $\nu$ values. Variability in $\nu$ appears to be due to variability among hosts (Dwyer et al. 1997), and so we assume in particular that there is a distribution of $\nu$ across hosts. This leads to the model

$$
\begin{aligned}
& \frac{d S}{d t}=-\bar{\nu}\left[\frac{S(t)}{S(0)}\right]^{C^{2}} S P, \\
& \frac{d P}{d t}=\bar{\nu}\left[\frac{S(t-\tau)}{S(0)}\right]^{C^{2}} P(t-\tau) S(t-\tau)-\mu P,
\end{aligned}
$$

where $\bar{\nu}$ and $C$ are the average and the CV, respectively, of the distribution of $\nu$, so that $C$ is a measure of heterogeneity in the attack rate. We have thus substituted $C^{2}$ for $\mathrm{CV}^{2}$, to avoid the confusion that arises from using multiple letters to refer to a single quantity. This model provides a much better description of NPV epidemics than equations (1) and (2), suggesting that heterogeneity in infection risk plays an important role in NPV transmission (Dwyer et al. 1997, 2002). To show how high heterogeneity in the attack rate produces a stable equilibrium, we extend this model to allow for multiple generations.

An important point is that the risk that insects become infected with an NPV is usually measured by exposing groups of larvae to a known dose of the virus and then observing the fraction that become infected (Watanabe 1987). Because larvae that do not consume the entire dose are discarded, such experiments effectively measure the risk of infection given exposure, which is usually thought of as susceptibility. In contrast, the transmission parameter $\nu$ allows for not just the risk of infection given exposure but also the risk of exposure. Variability in both types of risk can affect the overall risk that gypsy moth larvae become infected with the NPV (Dwyer et al. 2005), and so heterogeneity in $\nu$ provides a useful measure of variability in overall infection risk.

In previous work, Dwyer et al. (1997) attempted to measure heterogeneity in $\nu$ for the gypsy moth virus. The confidence intervals on the resulting estimates, however, were so large that it was impossible to determine which was more likely, a stable equilibrium or stable cycles (Dwyer et al. 2000). By refining experimental protocols (Dwyer et al. 2005) and collecting more data, here we are able to show that for this virus, values of heterogeneity $C$ are high enough to produce a stable equilibrium. The models thus appear to be making an incorrect assumption.

The data further suggest that infection risk declines after epidemics, whereas the models assume that infection risk is constant. We therefore constructed alternative models in which infection risk evolves because of natural selection, and these evolutionary models allow realistic cycles even with high heterogeneity. In the evolutionary models, infection risk declines after outbreaks because of selection for resistance, consistent with the data, and rises between outbreaks because of a fecundity cost of resistance. Experiments with full-sibling and half-sibling families provide preliminary evidence that infection risk is heritable, as assumed by the models. We therefore argue that natural selection on infection risk plays a role in gypsy moth outbreaks. Because the models are completely general and because pathogens play a key role in the dynamics of many outbreaking insects (Myers 1993; Moreau and Lucarotti 2007), we suggest that natural selection may play a role in outbreaks of other insects as well.

\section{The Standard Model: Infection Risk Is Constant}

\section{Constructing the Standard Model}

To construct the standard model, we first define $N_{t}$ and $Z_{t}$ as the initial host and pathogen densities in generation $t$, respectively. To connect the timescale of generations to the timescale of epidemics, we set $S(0) \equiv N_{t}$ and $P(0) \equiv Z_{t}$ in equations (3) and (4). Note that in $N_{t}, t$ denotes the discrete time of generations, while in $S(t)$, it instead denotes the continuous time of epidemics. Given these definitions, from equations (3) and (4) we can derive an implicit expression for the total fraction infected during the epidemic, $i\left(N_{t}, Z_{t}\right)$ (Dwyer et al. 2000):

$$
\left.1-i\left(N_{t}, Z_{t}\right)=\left\{1+\frac{\bar{\nu} C^{2}}{\mu}\left[N_{t} i\left(N_{t}, Z_{t}\right)+\eta Z_{t}\right)\right]\right\}^{-1 / C^{2}}
$$

Using this equation, we can show that the fraction infected increases rapidly with increasing host density and that the rate of this increase is reduced as heterogeneity is increased (Dwyer et al. 2000). Increasing heterogeneity thus reduces the strength of density dependence, specifically by increasing the number of hosts that have a low risk of infection.

To make the connection to long-term dynamics, we allow surviving hosts to reproduce, and we allow for between-generation survival of the virus (Dwyer et al. 2000): 
A.

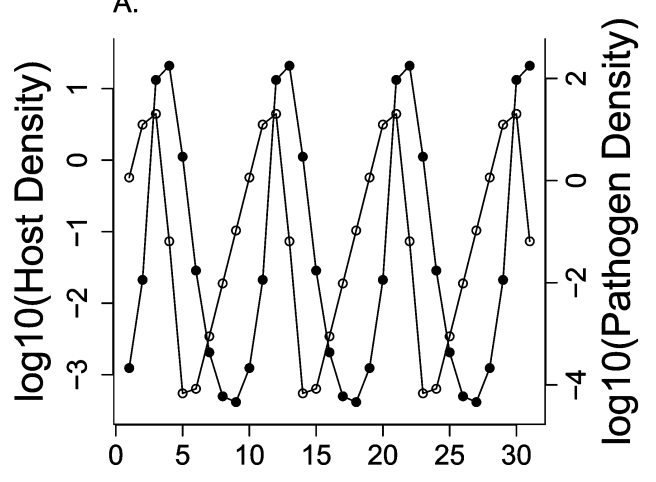

C.

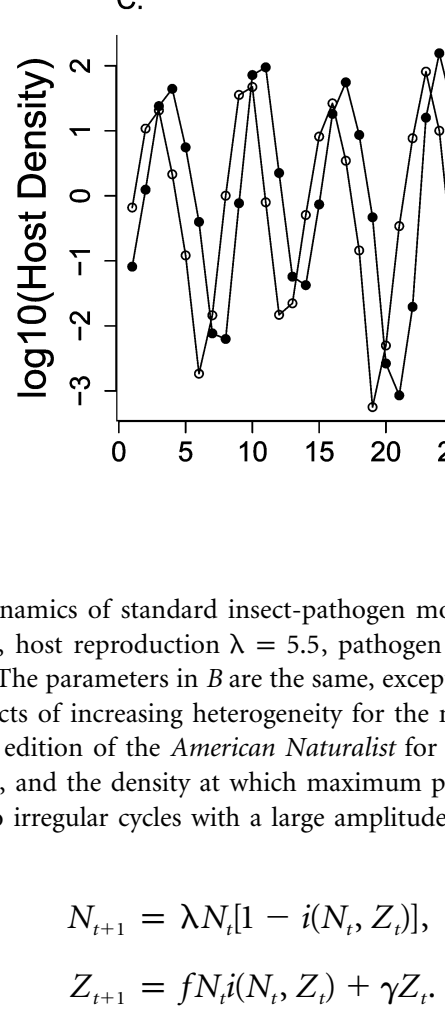

Here $f$ and $\gamma$ are the survival rates of pathogen particles produced in the most recent epidemic and in previous epidemics, respectively. We allow for the possibility of differences in the two survival rates because particles produced in the current epidemic are likely to have a greater chance of infecting larvae in the following generation (Murray and Elkinton 1989, 1990). Note that, in contrast, Anderson and May's (1980) well-known insect-pathogen model unrealistically assumes overlapping generations, which is why that model shows stable cycles even in the absence of heterogeneity in attack rates.

Although we tested the model using the gypsy moth $\mathrm{NPV}$, in fact the model applies to many different insectpathogen interactions. To illustrate this, we note that the
B.

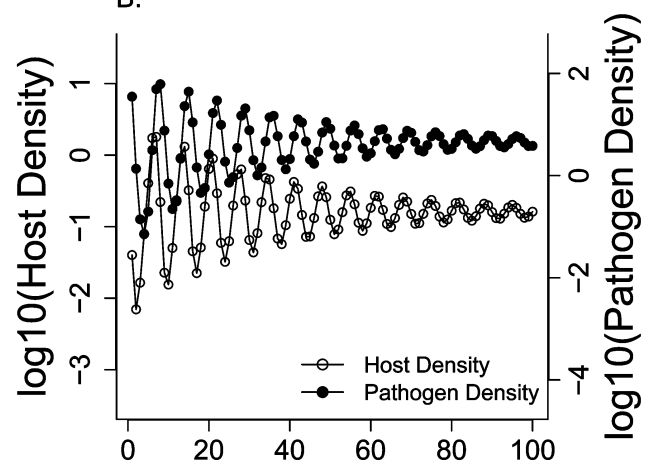

D.

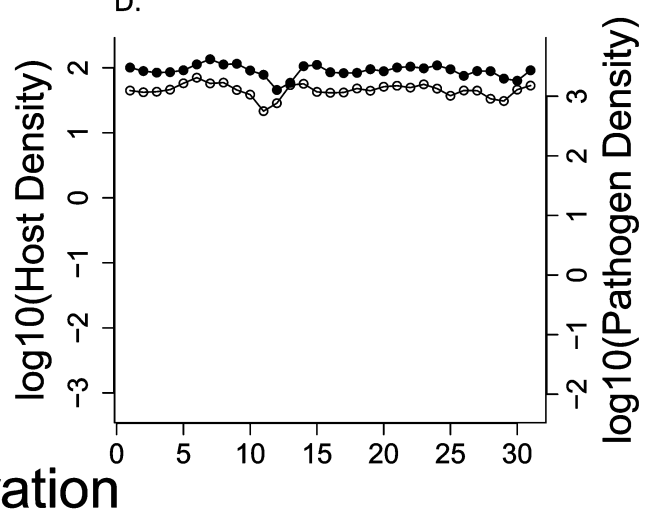

Figure 1: Dynamics of standard insect-pathogen models, which assume that infection risk is constant. $A$ and $B$ show the dynamics of equations (5)-(7). In $A$, host reproduction $\lambda=5.5$, pathogen between-generation impact $\phi=35$, and heterogeneity in susceptibility $C=0.86$, leading to the leading to damped oscillations and thus a stable equilibrium. $C$ and $D$ similarly rate $a=0.96$, and the density at which maximum predation occurs $b=0.14$ ), except that in $C, C=0.96$, while in $D, C=1.12$. For this model, $C<1$ leads to irregular cycles with a large amplitude, but $C>1$ produces only small-amplitude fluctuations about the equilibrium. and that it must survive in the environment between generations. Directly transmitted, fatal diseases that infect only larvae occur in a large number of insects. Pathogens with specialized stages that allow survival between host generations are also common among insects and include viruses, fungi, and protozoa (Fuxa and Tanada 1987; Miller 1997).

Among forest defoliators in particular, there are many species for which epidemics affecting the larval stages cause outbreaks to collapse (Myers 1993; Moreau and Lucarotti 2007), as in our models (Dwyer et al. 2000), and discrete generations are very common (Hunter 1991, 1995). Recent work by G. Dwyer, B. D. Elderd, and M. Coram (unpublished manuscript) has further suggested that heterogeneity in infection risk occurs among many forest insects 

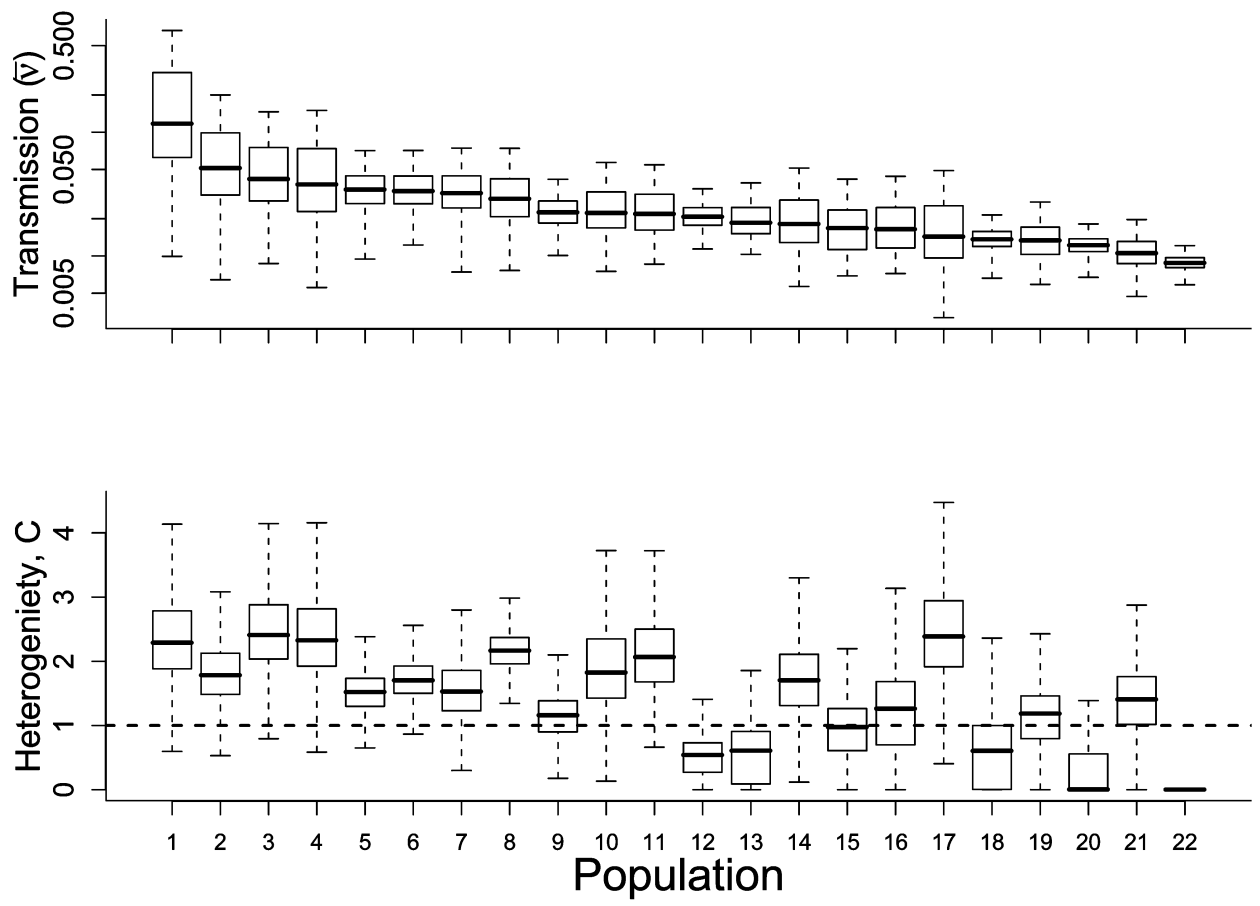

Figure 2: Transmission $\bar{\nu}$ and heterogeneity in susceptibility $C$ for each population in our transmission experiments. Populations are arranged in order of decreasing values of $\bar{\nu}$. This is a box-and-whisker plot, in which the boxes show the interquartile range, while the whiskers show the range of data values included within $1.5 \times$ the interquartile range. Values of heterogeneity $C$ were thus usually $>1$, but values of transmission $\bar{\nu}$ varied greatly.

and their NPVs. By using a general model, we are thus attempting to make a general argument about insect-pathogen interactions.

The model unites two timescales: that of weeks over which epidemics occur and that of generations over which outbreaks occur. We can therefore understand the model's long-term behavior in terms of the dynamics of epidemics. First, increases in transmission lead to increases in epidemic severity, and so increases in $\bar{\nu}$ reduce the equilibrium density of hosts (Dwyer et al. 2000). By reducing the severity of density dependence, increases in $C$ instead reduce the propensity to cycle, such that cycles are possible only if $C<1$ (fig. $1 A$ ); $C>1$ or, equivalently, $\mathrm{CV}^{2}>1$, guarantees a stable equilibrium (fig. $1 B$ ). When cycles do occur, they are extremely regular even if we add stochasticity, a behavior that does not match data on outbreaking insects (not shown; see Dwyer et al. 2004). If we also add generalist predators (see the appendix in the online edition of the American Naturalist; also see Dwyer et al. 2004), we obtain realistic, irregular cycles when $C<1$ (fig. 1C), but when $C>1$, only small-amplitude fluctuations occur (fig. $1 D$ ).

\section{Quantifying Heterogeneity in Infection Risk}

Methods. To test the standard model, equations (5)-(7), we estimated $C$, the heterogeneity among gypsy moth lar- vae in their risk of becoming infected with the NPV. As we have described, the standard approach to quantifying infection risk with NPVs is to use laboratory dose-response experiments, in which larvae are fed a range of viral doses and larvae that do not consume the entire dose are discarded (Watanabe 1987). Infection risk is then measured in terms of the $50 \%$ lethal dose. Dose-response experiments thus measure the probability of infection given virus consumption, rather than the overall probability of infection and so do not allow for the effects of host feeding behavior. Variability in host feeding behavior, however, can have strong effects on infection risk (Capinera et al. 1976; Dwyer et al. 2005) and may provide a more variable trait for selection to act on than susceptibility per se (B. Parker, B. D. Elderd, and G. Dwyer, unpublished manuscript). Moreover, relating infection risk to the dynamics of outbreaks is difficult without mathematical models, and the models require an estimate of the overall probability of infection. More precisely, the models require estimates of $\bar{\nu}$, the average probability of infection per cadaver and per unit time, and the coefficient of variation $C$.

We thus depart from standard practice by estimating overall infection risk. Our approach is to allow uninfected larvae to feed on virus-contaminated foliage in the field, across a range of virus densities (appendix; also see Dwyer 

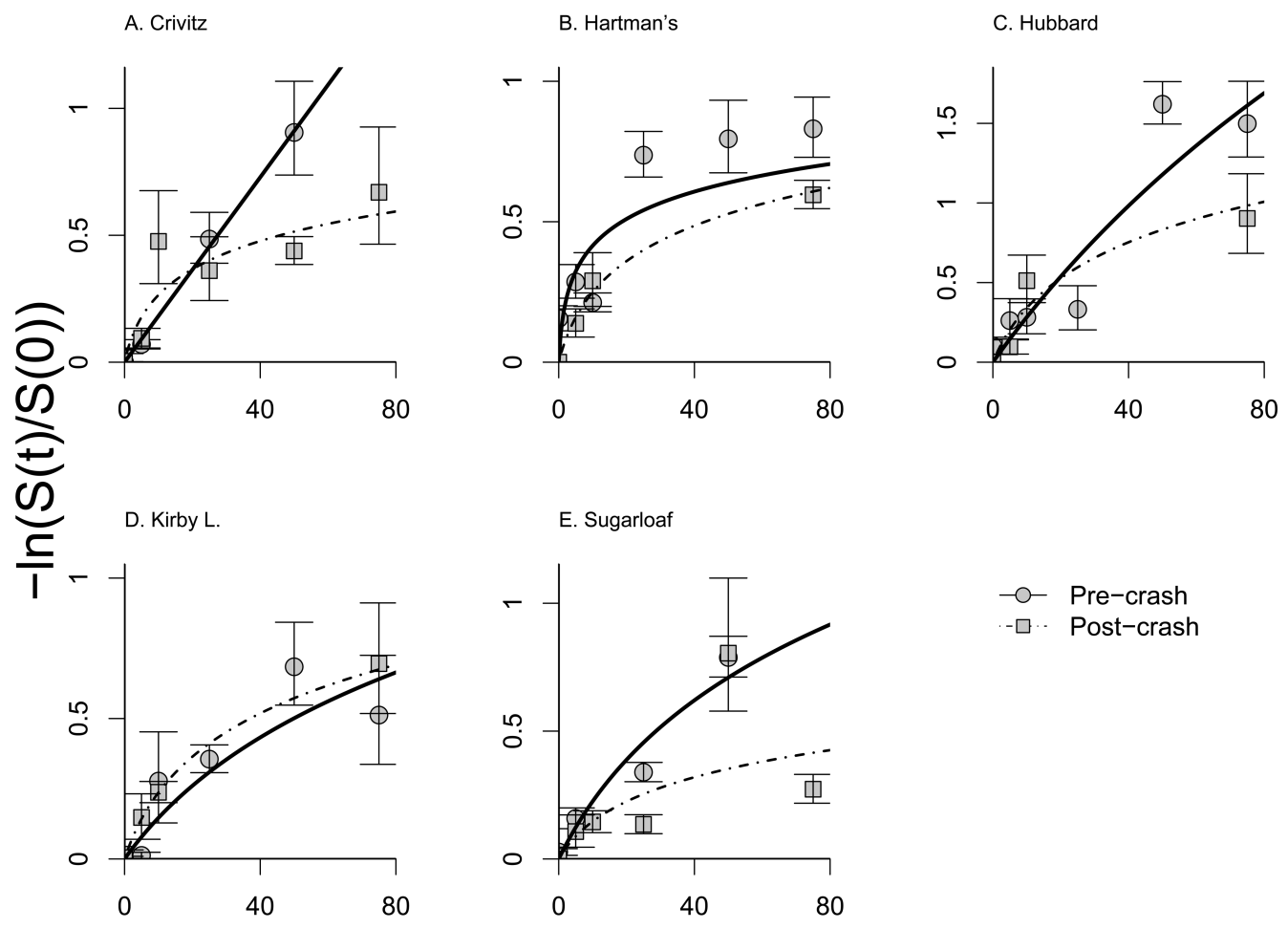

Figure 3: Experimental measurements of infection rates before and after population crashes in five gypsy moth populations. Points represent data, and lines represent the best fit of equation (8) in each population in each year. Here we plot the log-transformed fraction uninfected, $-\ln (S(T) / S(0)$ ), which makes it easier to detect changes in transmission parameters. Although the results vary across populations, infection risk was generally lower after crashes. Note that changes in the shape of the best-fit model are due to changes in heterogeneity $C$ (Dwyer et al. 1997).

and Elkinton 1993; Dwyer et al. 1997, 2005; D’Amico et al. 1998). Like dose-response experiments, these experiments include a range of viral doses, but they also allow for realistic feeding behavior, and they can be used to estimate $\bar{\nu}$ and $C$. Indeed, estimates of $\bar{\nu}$ and $C$ from these experiments produce model predictions that are close to the dynamics of natural epidemics (Dwyer et al. 2002), suggesting that transmission rates in our experiments are not that different from those in nature.

To reduce the uncertainty in our estimates of $\bar{\nu}$ and $C$, we followed Dwyer et al. (2005) in instituting several improvements over previous experiments (Dwyer et al. 1997). The most important improvement is that we used larvae that had all reached the fourth instar, or stage, within 24 $\mathrm{h}$, thereby avoiding the changes in susceptibility that occur within the first few days of the instar (Grove and Hoover 2007). Given this change, we again allowed the initially uninfected larvae to feed for a week in the field, and then we reared them in the lab to see which of them had become infected while in the field. To estimate $\bar{\nu}$ and $C$, we fitted the epidemic model to the resulting data. Because in our experiments branches were enclosed in mesh bags that prevent the breakdown of the virus (G. Dwyer, unpublished data), we can set $d P / d t=0$ in equations (3) and (4), which allows us to solve equation (3) as follows (Dwyer et al. 1997):

$$
\frac{S(T)}{S(0)}=\left(1+\bar{\nu} C^{2} P(0) T\right)^{-1 / C^{2}} .
$$

Here $T$ is the length of time for which an experiment ran, so that $S(T)$ and $S(0)$ are the densities of uninfected larvae at the end and the beginning of the experiment, respectively, and $P(0)$ is the initial density of virus, in the form of infectious cadavers; $S(T) / S(0)$ is thus the fraction uninfected at the end of the experiment, and $\bar{\nu}$ and $C$ can then be estimated by fitting equation (8) to the data, using maximum likelihood (Pawitan 2001) and nonlinear fitting routines (Venables et al. 2005; also see appendix).

To assess variability over space and time, we used test larvae reared from egg masses collected over 4 years across a wide area of the northeastern and midwestern United 

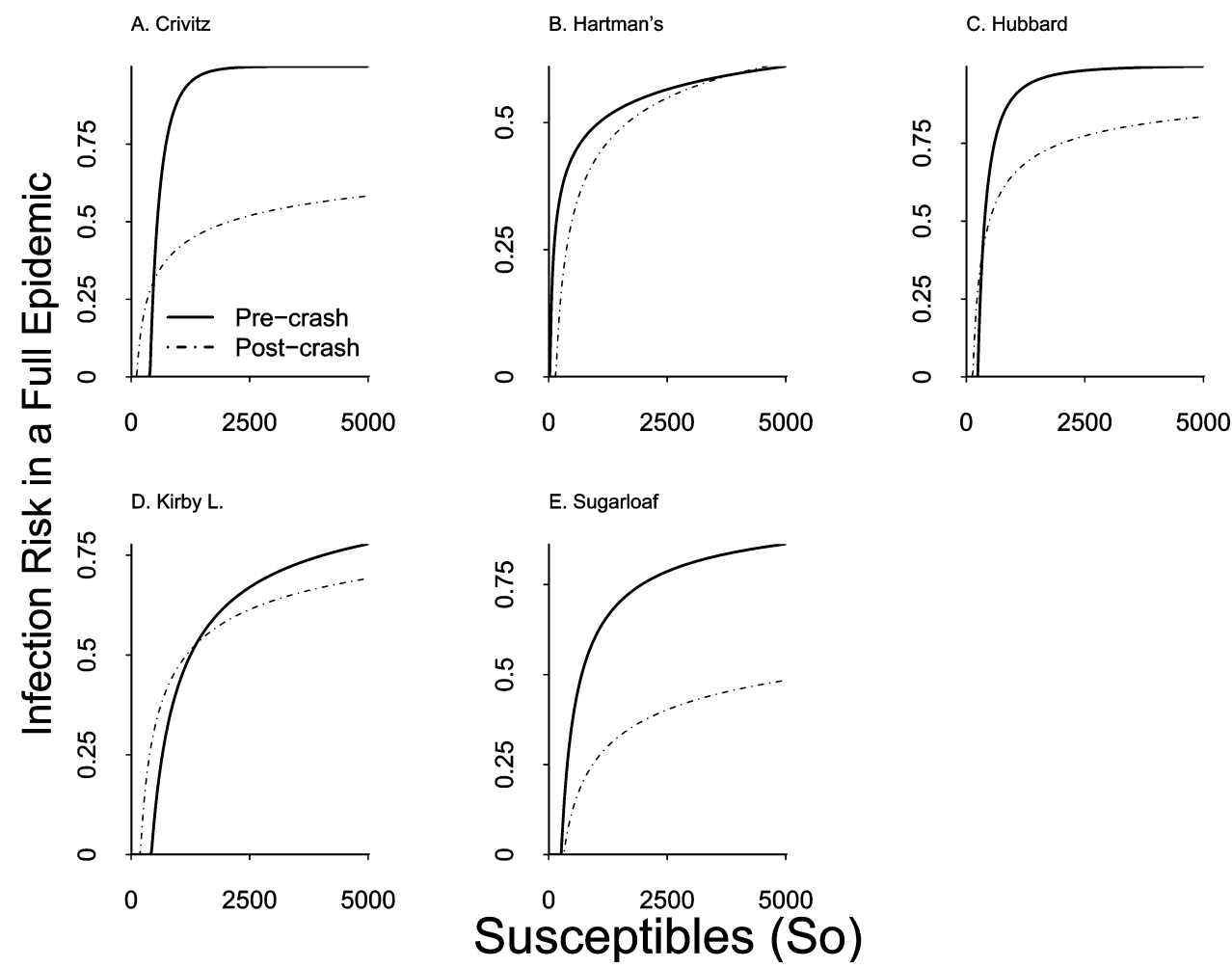

Figure 4: Consequences of the changes in infection rates shown in figure 3 for infection risk in a full epidemic, $i\left(N_{t}, Z_{t}\right)$, as calculated from equation (5). Because changes in heterogeneity $C$ modulate the effects of density, we considered a range of initial host densities $N_{t}$, but for simplicity, we assumed a constant initial infection rate of $5 \%$ (Woods and Elkinton 1987). In general, infection risk was reduced in most populations at most densities, suggesting that infection risk is reduced after population crashes.

States (table A1 in the online edition of the American Naturalist). Egg masses were collected from areas of a few square kilometers or less within larger forests of tens to hundreds of square kilometers. Each collection site, and thus each population, was at least $50 \mathrm{~km}$ from all other sites, a distance sufficient to ensure that migration was low (Dwyer and Elkinton 1995). Although gypsy moth outbreaks are roughly synchronous over large areas (Williams and Liebhold 1995; Peltonen et al. 2002), our populations were far enough apart that crashes often occurred in different years. As often as possible, we collected from the same populations during the pre- and postcrash phases of the population cycle, but mortality during crashes was so severe that we were able to do so in only five cases.

Results. Figure A1 in the online edition of the American Naturalist shows the data and the best-fit version of the transmission model, equation (8). In all cases, a $\chi^{2}$ goodness-of-fit test could not reject the model (table A1). Estimates from this model of average transmission $\bar{\nu}$ and heterogeneity $C$ for each population in each year are shown in figure 2. In 16 of 22 cases, the point estimate of $C$ was greater than 1, and heterogeneity in infection risk was thus usually high enough to guarantee a stable equilibrium in the long-term model. Because a stable equilibrium cannot be reconciled with data on gypsy moth outbreaks (Johnson et al. 2005), the data therefore reject the long-term models.

Figure 2 also shows that the average transmission parameter $\bar{\nu}$ varied by as much as an order of magnitude across populations and years. This variability cannot be explained by spatial structure or by latitude (appendix), but some of it can be explained by changes over time. For the five populations for which we had both pre- and postcrash measurements, infection risk generally declined after crashes (fig. 3), and Akaike Information Criterion (AIC) analysis confirmed that there were effects of year on both $\bar{\nu}$ and $C$ (AIC results and parameter values in tables A3, A4, respectively, in the online edition of the American Naturalist). The data thus suggest that the incorrect assumption of the standard models is that infection risk is constant.

Although the data in figure 3 generally show strong changes in infection risk, the best-fit model often changed from year to year in both initial slope and overall shape. 


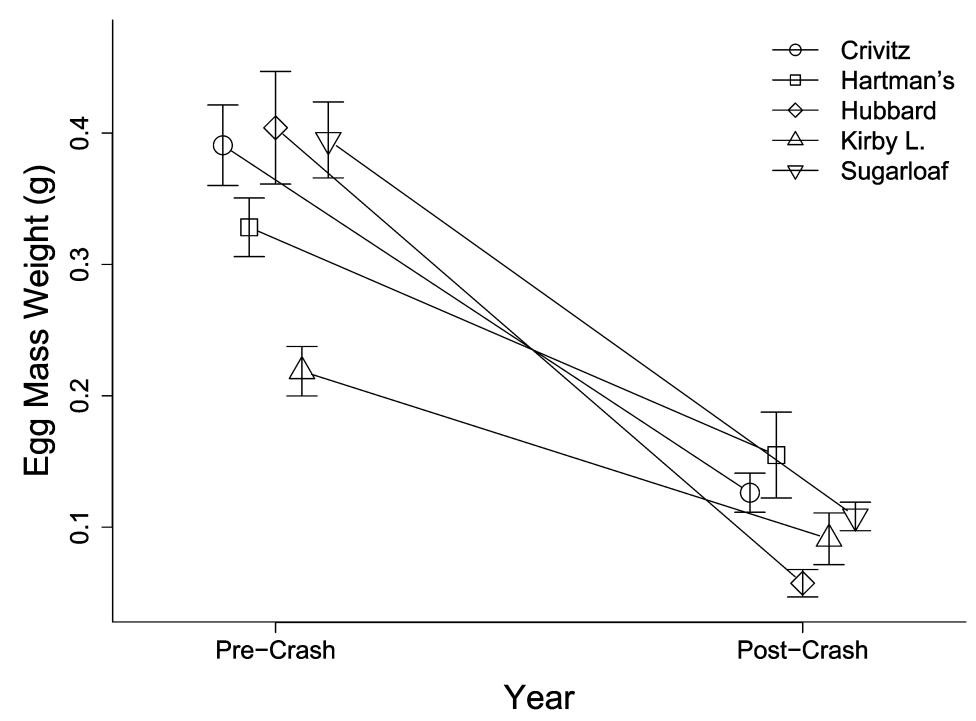

Figure 5: Average egg mass weights with $95 \%$ confidence intervals for the five gypsy moth populations from which we collected egg masses before and after population crashes. For clarity, data points are slightly displaced horizontally. All differences within populations are significant $(P<.01$, Wilcoxon test).

The complexity of these changes makes it difficult to see what the data imply for epidemics. We therefore inserted each pair of parameter estimates into equation (5) to see how epidemic severity $i\left(N_{t}, Z_{t}\right)$ changed over time in each population. Figure 4 then shows that at most densities in most populations, $i\left(N_{t}, Z_{t}\right)$ was lower after the crash than before the crash. These changes in infection risk were quite variable among populations, but with only five cases, we are not yet in a position to fully explain the causes of the variability.

\section{An Evolutionary Model: Natural Selection Drives Fluctuations in Infection Risk}

\section{Constructing the Evolutionary Model}

Our data thus reject the models in which infection risk is constant and instead suggest that infection risk declines after outbreaks. A simple explanation for this pattern is that natural selection imposed by the pathogen led to increased resistance. We therefore extended the standard host-pathogen models to allow for natural selection. Arguably, models in which natural selection drives changes in infection risk are more biologically reasonable than models in which infection risk is constant. Note that the epidemic model, equations (3) and (4), predicts that average transmission $\bar{\nu}$ declines as the epidemic progresses because the more susceptible hosts are removed more rapidly from the population (Dwyer et al. 2000). In assuming that infection risk is constant, the standard models are thus assuming that reproduction causes average susceptibility to return to the value that it had before the epidemic. Variability in infection risk across hosts is therefore assumed to be determined entirely by environmental factors, even though equations (3) and (4) assume that an individual's risk is constant across its lifetime. These assumptions are hard to justify biologically.

In altering the standard models, we therefore assumed that infection risk is heritable. All else being equal, infection risk would then decline to the lowest achievable value. Because this is clearly contradicted by our data, we followed previous models of host evolution in assuming that there is a fecundity cost of resistance (Gillespie 1975). Our observational data provide at least circumstantial evidence of a cost of resistance, in that egg mass weights consistently declined after crashes (fig. 5). These reductions, however, may also have been due to food limitation, which occurs during outbreaks because of defoliation (Elkinton and Liebhold 1990), or to sublethal infections, which have been shown to occur among gypsy moths in the lab (Myers et al. 2000). We thus do not have direct experimental evidence of costs of resistance in gypsy moths, but there is strong evidence of costs of resistance to NPVs among other insects (Fuxa and Richter 1998; Lee et al. 2006; Mealor and Boots 2006). The mechanisms underlying these costs are generally unknown, but for NPVs in particular, there are at least two candidate mechanisms that could produce a cost. First, NPVs infect insects by attaching to receptors on the insect gut (Horton and Burand 1993), and the loss of these receptors would presumably lead to both reduced 
A.

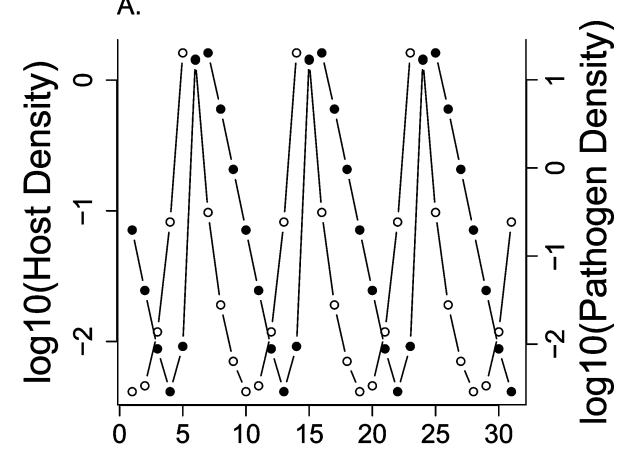

C.

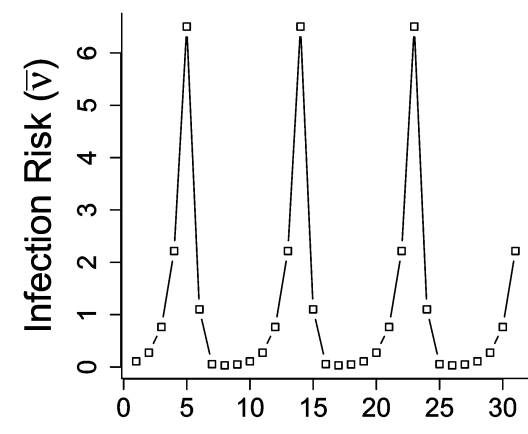

B.

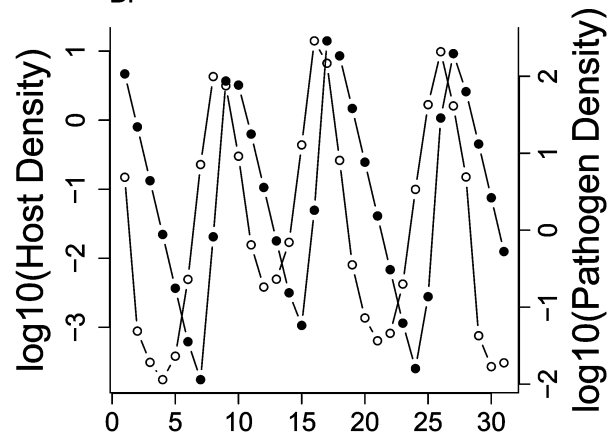

D.

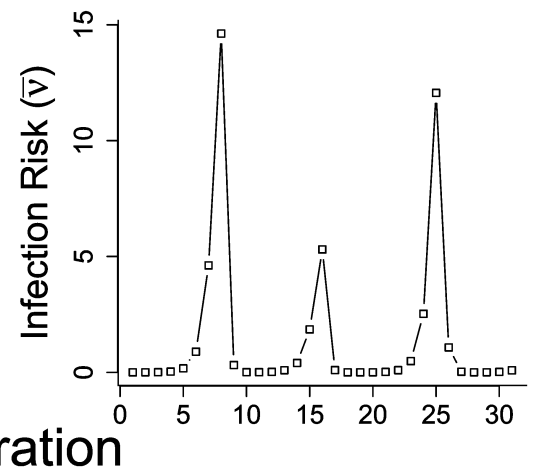

Figure 6: Dynamics of host-pathogen models in which natural selection drives fluctuations in infection risk. A shows host and pathogen densities for the host-pathogen-only model, equations (9)-(12), while $B$ shows densities for the corresponding host-pathogen-predator model, which includes stochasticity and a generalist predator by substituting equation (A22) in the online edition of the American Naturalist for equation (9). C and $D$ show the corresponding fluctuations in the average transmission rate for each model to illustrate that average transmission rises and falls in synchrony with host density. In $A$ and $C$, baseline reproduction $r=0.2$, rate of increase of reproduction with increasing susceptibility $\lambda=9$, pathogen longterm survival $\gamma=0.2$, heterogeneity in susceptibility $C=2$, and pathogen between-generation impact $\phi=14$. In $B$ and $D$, the parameters are the same as in $A$ and $C$, with the additions that the maximum fraction of prey consumed $a=0.967$ and the density at which predation is maximized $b=0.14$. Note that $B$ and $D$ show irregular fluctuations, as in insect outbreaks in nature (Dwyer et al. 2004).

susceptibility and reduced digestive efficiency and thus to a cost of resistance. Second, gypsy moth larvae in particular are known to avoid anything on a leaf surface that has the consistency of a virus-infected cadaver, including not just cadavers but also molasses (Capinera et al. 1976). Ongoing work in G. Dwyer's lab has suggested that this trait may cause larvae to reject leaf tissue simply because of leaf scars (L. Eakin and G. Dwyer, unpublished data). Increases in the sensitivity of the trait might therefore reduce both infection risk and feeding efficiency, thereby leading to reduced egg mass size and thus a cost of resistance.

In short, it seems reasonable to allow for a cost of resistance. Also, for simplicity, we assume that heterogeneity $C$ is constant and that offspring have the same phenotype as their parents, so that we ignore sexual reproduction. These assumptions lead to the following model (see appendix):

$$
\begin{gathered}
N_{t+1}= \\
N_{t}\left[1-i\left(N_{t}, Z_{t}, \bar{\nu}_{t}\right)\right]\left\{r+\lambda \bar{\nu}_{t}\left[1-i\left(N_{t}, Z_{t}, \bar{\nu}_{t}\right)\right]^{C^{2}}\right\} \\
Z_{t+1}=f N_{t} i\left(N_{t}, Z_{t}, \bar{\nu}_{t}\right)+\gamma Z_{t} \\
\bar{\nu}_{t+1}= \\
\frac{r \bar{\nu}_{t}\left[1-i\left(N_{t}, Z_{t}, \bar{\nu}_{t}\right)\right]^{C^{2}}+\lambda\left(C^{2}+1\right) \bar{\nu}_{t}^{2}\left[1-i\left(N_{t}, Z_{t}, \bar{\nu}_{t}\right)\right]^{2 C^{2}}}{r+\lambda \bar{\nu}_{t}\left[1-i\left(N_{t}, Z_{t}, \bar{\nu}_{t}\right)\right]^{C^{2}}} \\
1-i\left(N_{t}, Z_{t}, \bar{\nu}_{t}\right)= \\
\left\{1+\frac{\bar{\nu}_{t} C^{2}}{\mu}\left[i\left(N_{t}, Z_{t}, \bar{\nu}_{t}\right) N_{t}+\eta Z_{t}\right]\right\}
\end{gathered}
$$

Here $r$ is the fecundity of individuals that are completely resistant $(\nu=0)$, so that $r+\lambda \nu$ is the reproductive rate 


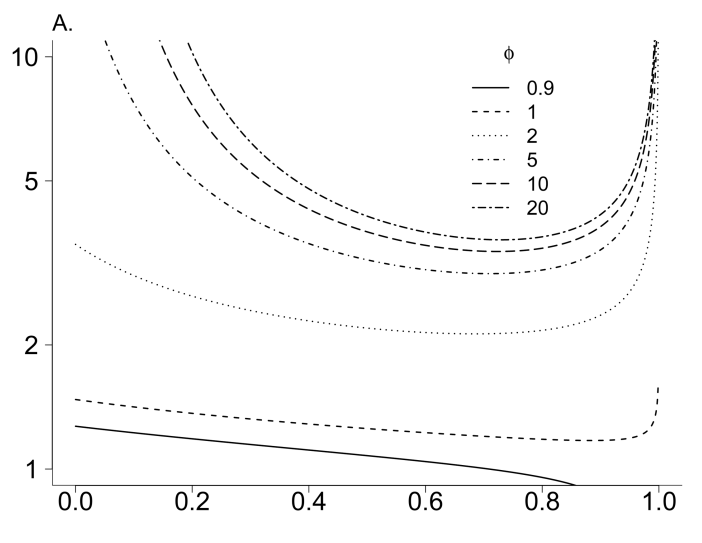

\section{Stable Point Equilibrium $\uparrow$ Limit Cycles $\downarrow$}
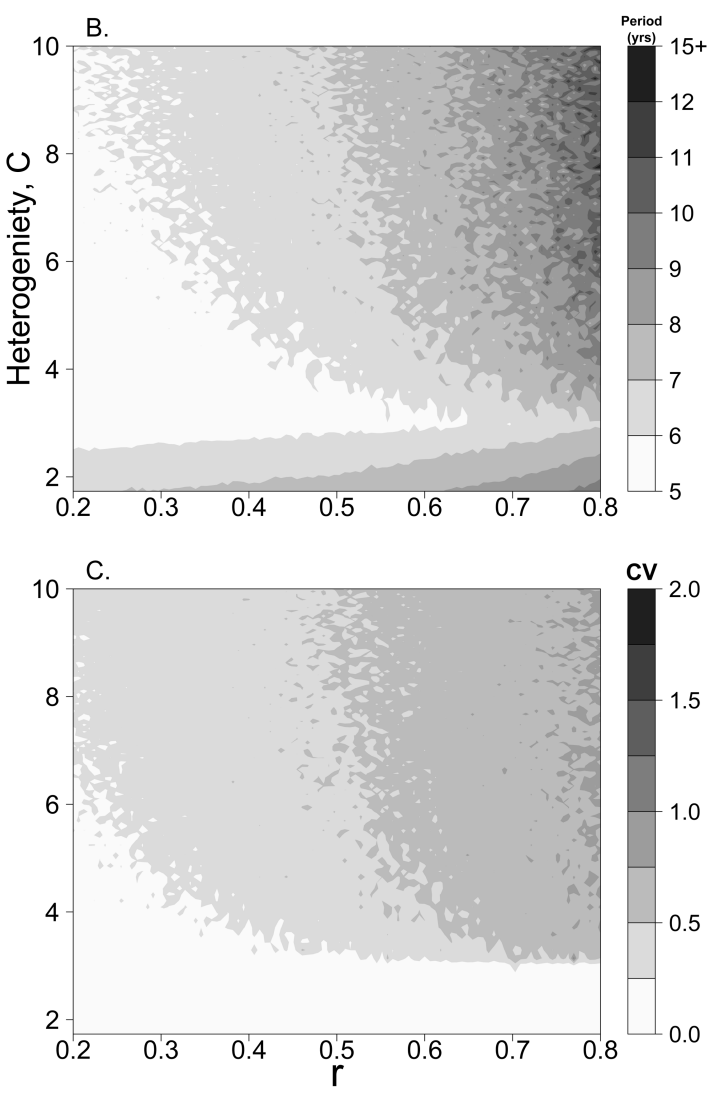

Figure 7: Range of parameter values for which cycles occur for the models in which infection risk is affected by natural selection. $A$, Host-pathogen model, equations (9)-(12). Each line represents the boundary between cycles and stability for different values of pathogen between-generation impact $\phi$, such that limit cycles occur for values of heterogeneity $C$ below each line. There is thus a large region of parameter space for which cycles occur, even for $C>1$. B, Time between outbreaks, averaged over 100 realizations, for the host-pathogen-predator model with natural selection, equations (10)-(12) and (A22) in the online edition of the American Naturalist, with $\phi=5$. C, Coefficient of variation (CV) of time between outbreaks for the host-pathogen-predator model with natural selection and $\phi=5$. For gypsy moth outbreaks in nature, the average time between outbreaks is 6-10 years (Johnson et al. 2005), with CV values between 0.2 and 0.7 (Dwyer et al. 2004). The host-pathogen-predator model thus produces realistic cycles for a wide range of parameter values. 
A.
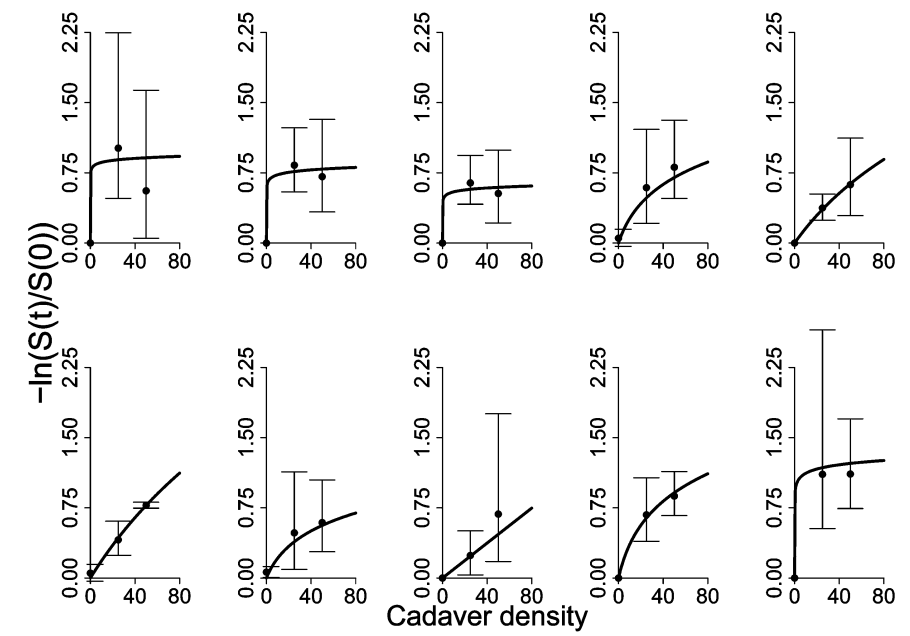

B.

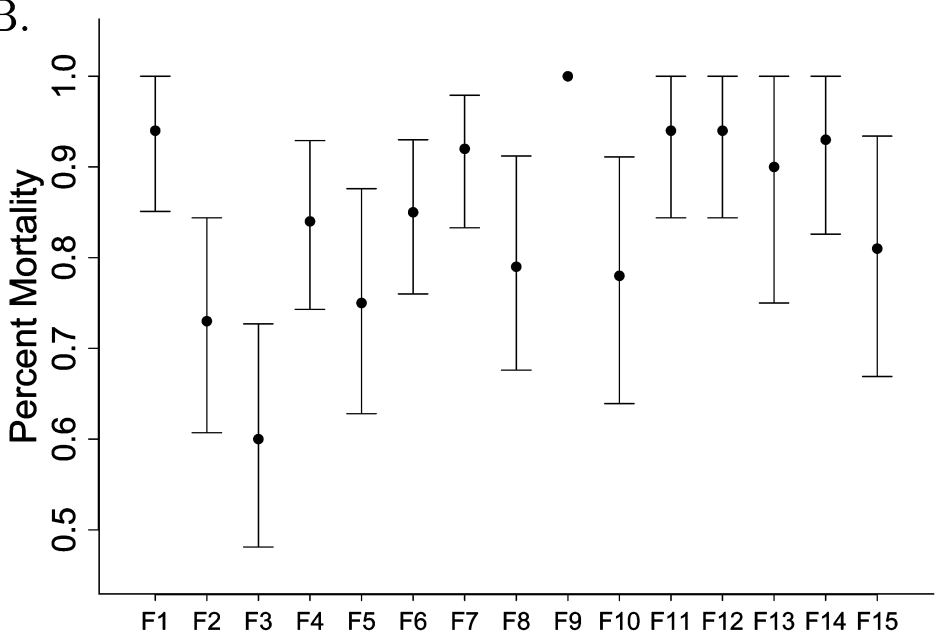

Family

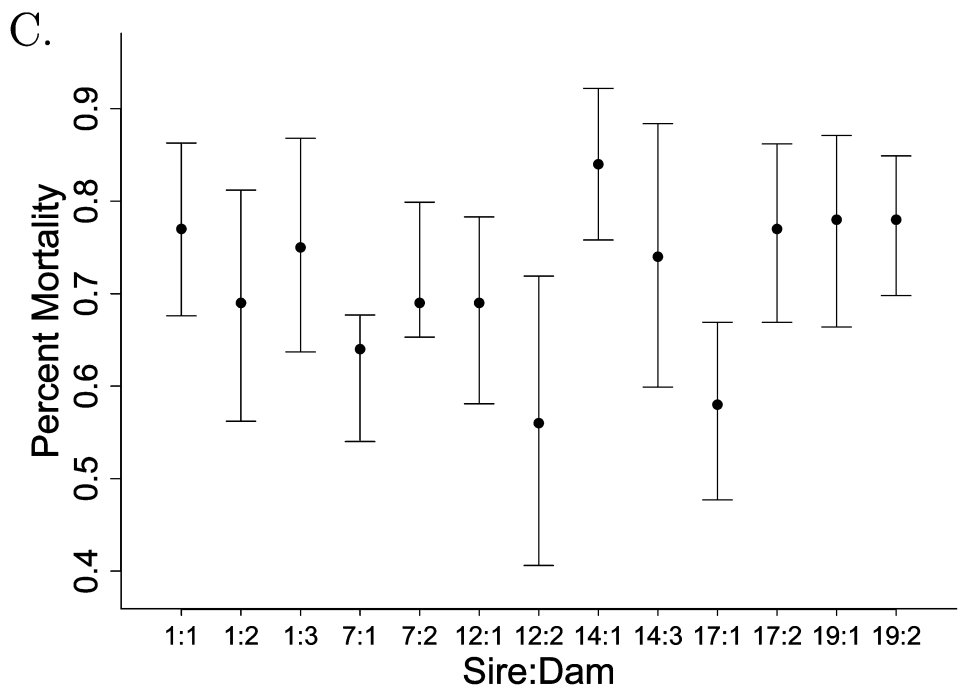


of individuals with transmission parameter $\nu$. The key difference from equations (5)-(7) is thus that average transmission is now a dynamic variable, $\bar{\nu}_{t}$.

For this model, we require first that $r<1$ because, otherwise, completely resistant hosts increase without bound (appendix). Long-period, large-amplitude cycles then occur even for $C>1$ (fig. 6). As figure 6 shows, in this model repeated epidemics cause average transmission $\bar{\nu}_{t}$ to drop sharply after outbreaks. The fecundity cost of resistance then causes transmission to slowly rise between outbreaks, until rising virus levels again lead to strong selection for resistance. Cycles therefore occur even when $C$ is high because changes in average transmission reduce the stabilizing effect of highly resistant individuals.

This model thus reconciles our experimental data with the occurrence of cycles. More quantitatively, our estimates of heterogeneity $C$ produce cycles in the evolutionary model for a wide range of values of the other parameters (fig. 7A). Moreover, if we allow for both predation and stochasticity by substituting equation (A22) in the online edition of the American Naturalist for equation (9), the average (fig. $7 \mathrm{~B}$ ) and the $\mathrm{CV}$ (fig. $7 \mathrm{C}$ ) of the time between outbreaks are close to data from real populations (Dwyer et al. 2004; Johnson et al. 2005).

The model is also consistent with the declines in infection risk that we observed after epidemics (fig. 3). As our AIC analyses make clear, however (table A3), the data suggest that both $\bar{\nu}$ and $C$ change over time, even though the model allows for changes only in $\bar{\nu}$. Because allowing $C$ to change over generations leads to an extremely complicated model, in comparing the model to the data we instead reanalyze the data, assuming that $C$ is constant but that $\bar{\nu}$ changes over time. Table A5 in the online edition of the American Naturalist then shows that, in four cases out of five, average transmission declined over time, as predicted by the model. Although there were clearly sources of variability that acted on the data that are not accounted for by the model, the model with natural selection is nevertheless best able to reconcile our experimental data with the occurrence of outbreaks.

\section{Testing Whether Infection Risk Is Heritable}

Methods. A crucial untested assumption of the evolutionary models is that infection risk is heritable. In 2006 and
2007, we therefore carried out additional experiments to test for family effects on infection risk and thus to test for heritable variation. To do this, we reared larvae from individual egg masses in family groups, using full-sibling groups in two experiments and half-sibling groups in a third experiment. The distinction here is that in our experiments from 2000 to 2003, larvae from a given population were reared from $25-50$ egg masses mixed together, whereas in our 2006 and 2007 experiments, larvae from a given egg mass were reared separately from larvae from other egg masses. Mixing egg masses provided more larvae and thus more replicates per population, which in turn allowed more accurate estimates of heterogeneity $C$. In contrast, rearing larvae in family groups from individual egg masses did not permit us to estimate parameters as accurately, but it allowed us to test directly for effects of family on virus transmission.

In the case of the full-sibling experiments, we hatched larvae from separate egg masses and reared them in fullsibling groups. In the case of the half-sibling experiment, we again reared larvae in full-sibling groups, but then we mated males to each of two or three females, with each female being mated only once. These mated females then produced eggs and thus larvae in half-sibling groups for our experiments. This design allowed us to test for effects of sire as distinct from effects of dam and thus to separate genetic effects from maternal effects.

Results. In all cases, transmission varied strongly between families (fig. 8), and AIC analyses confirmed that models with no family effects provide very poor explanations for the data (tables A6, A7 in the online edition of the American Naturalist). For the half-sibling data in particular (table A8 in the online edition of the American Naturalist), a model with a sire effect provides the best explanation for the data, suggesting that infection risk has a genetic component and is thus not simply due to maternal effects. Nevertheless, models that include dam effects explain the data nearly as well, and so it seems likely that both sire and dam affect infection risk.

From these data, we were also able to calculate heritabilities, following Bull et al. (1982). Broad-sense heritabilities from the full-sibling experiments were 0.22 and 0.42 , respectively (for 2006 , we used only the 50 -cadaver

Figure 8: Results of transmission experiments with full- or half-sibling larvae. A shows data for full-sibling larvae from 2006. Each panel shows infection rates for a different family, with points representing the data and lines representing the best-fit version of equation (8). $B$ shows data for full-sibling larvae in 2007, and $C$ shows data for half-sibling larvae in 2007. In 2007, we used only one virus density, and so each point in $B$ and $C$ represents data for a different group. On the horizontal axis in $C$, at each tick mark, the number before the colon is the sire number, while the number after the colon is the sire-specific dam number; 12:2 thus refers to larvae produced by the second dam that was mated to the twelfth sire. All dams were mated to only one sire. For logistic reasons, the number of groups varied among experiments, but in all cases each treatment was replicated eight times. 
treatment, but results were similar for the 25-cadaver treatment), while the narrow-sense heritability from the halfsibling experiment was 0.14 . While these heritabilities are modest, we note that the founding egg masses for these experiments were all collected from a single population, and in the half-sib experiment in particular, for logistic reasons, we were able to use only six sires. Under these circumstances, we did not expect high heritabilities.

Because the data thus do not conclusively demonstrate that infection risk is heritable, it could be that the changes in infection risk in figure 3 were due to maternal effects instead of natural selection. To test whether changes in infection risk driven by maternal effects could lead to cycles, we constructed an alternative model in which fluctuations in transmission rate are driven by maternal effects. The underlying assumption of this model is that food limitation among mothers or grandmothers leads to reductions in infection risk among offspring (Ginzburg and Taneyhill 1994). The equation for the rate of change of transmission $\bar{\nu}_{t}$ is therefore based on the maternal-effects model of Ginzburg and Taneyhill (1994; model equations given in the appendix), so that population quality is translated directly into average susceptibility.

Surprisingly, this model does not permit cycles for heterogeneity $C>1$ (appendix). This difference from the evolutionary models probably occurs because population quality has a maximum value, as seems reasonable for a trait determined by resource availability (Ginzburg and Taneyhill 1994). In contrast, there is no obvious reason why infection risk should approach a maximum, and so in our evolutionary models, we do not include such a constraint. A rigorous test of these assumptions, however, is beyond the scope of our research. Accordingly, for now we note simply that we do not yet have a maternal-effects model that can reconcile high levels of heterogeneity in infection risk with the occurrence of outbreaks.

\section{Discussion}

Our experimental data clearly reject standard models of insect-natural enemy interactions and instead support models in which natural selection drives changes in infection risk. For gypsy moth larvae, values of heterogeneity in transmission $C$ are clearly high enough to produce stable equilibria in the models with constant infection risk (fig. 2 ), but the same values produce realistic cycles in models with natural selection (figs. 6, 7). The data also show that infection risk declines after population crashes (figs. 3, 4), consistent with the predictions of the evolutionary models, and they provide preliminary support for the model assumption that infection risk is heritable (fig. 8).

We recognize, however, that our models neglect other important features of the biology of North American gypsy moth populations. Gypsy moth outbreaks are also affected by food limitation (Abbott and Dwyer 2007), by the fungal pathogen Entomophaga maimaiga (Hajek 1999), and by interactions between the NPV and induced changes in foliage chemistry (Schultz and Baldwin 1982; Hunter and Schultz 1993). The latter effect in particular may provide an alternative mechanism by which outbreaks can be reconciled with high heterogeneity in transmission (B. D. Elderd, B. Rehill, and G. Dwyer, unpublished manuscript). Nevertheless, our goal is not to construct the ultimate model of insect outbreaks but instead to argue that models in which natural selection drives infection risk provide a better explanation for the data than do models with constant infection risk. Indeed, the standard models have been used to show that some heterogeneity in infection risk is necessary to prevent unstable cycles (Dwyer et al. 2000) and that generalist predators play an important role in producing irregular cycles (Dwyer et al. 2004). Both conclusions also hold for the models with natural selection. Extending models to allow for additional realism thus does not always change the conclusions of the models.

A key assumption of our models is that infection risk is heritable, but our data do not conclusively rule out the possibility that infection risk is instead determined by maternal effects. The larvae in our half-sibling experiments, however, represented the second generation of larvae reared in the lab, and the persistence of maternal effects over two generations seems unlikely. Likewise, a model in which changes in infection risk are driven by maternal effects does not produce cycles for high heterogeneity. Finally, early evidence of maternal effects in gypsy moths, mostly from laboratory experiments (Rossiter 1991a, $1991 b$ ), has been followed by recent experiments showing that such effects are weak or nonexistent in the field (Myers et al. 1998; Erelli and Elkinton 2000). Nevertheless, with enough additional data, we may yet discover that maternal effects also play a role. More generally, although measuring transmission parameters allowed us to directly test the models, it did not allow us to identify the traits that underlie variability in infection risk. Recent work by B. Parker, B. D. Elderd, and G. Dwyer (unpublished manuscript), however, has identified feeding behaviors that appear to play a key role in the evolution of infection risk. This work may provide us with a trait that can be measured on individuals, which should make it easier to test for heritability in infection risk.

Although our experiments used the gypsy moth, we emphasize again that our models are intended to be widely applicable to insect outbreaks and may apply to insectpathogen interactions in general. Indeed, given that most outbreaking insects have discrete generations (Hunter 1991, 1995), heterogeneity in infection risk is likely to play a key role for the many outbreaking insects whose dy- 
namics are driven by pathogens (Moreau and Lucarotti 2007). Work by G. Dwyer, B. D. Elderd, and M. Coram (unpublished manuscript) has shown that, in fact, values of heterogeneity $C$ estimated from epidemic data are also $>1$ for the Douglas fir tussock moth Orgyia pseudotsugata (Shepherd et al. 1984; Otvos et al. 1987) and the western tent caterpillar Malacosoma californicum (Kukan and Myers 1999). Our evolutionary models are therefore likely to provide a better explanation than standard models for the dynamics of these two insects. The same project has also shown that estimates of heterogeneity from gypsy moth epidemics are close to the estimates from our experiments, suggesting that the experimental estimates are not due to artifacts.

Because our models with natural selection have survived multiple tests, we argue that natural selection for host resistance plays a role in some insect outbreaks (Box 1979). This result is interesting first because it adds to a growing literature suggesting that the evolution of host resistance may have a strong effect on the population dynamics of host-pathogen interactions (Thrall and Antonovics 1995; Duffy and Sivars-Becker 2007). Second, outbreaking insects may provide an unusual example of an organism in which natural selection drives complex population dynamics (Saccheri and Hanski 2006). Third, the economic importance of forest insects (Liebhold et al. 2000) and the usefulness of pathogens in controlling these insects (Hunter-Fujita et al. 1998; Moreau and Lucarotti 2007) together suggest that natural selection may be relevant for forest pest management.

Finally, an important feature of our work is that it suggests that the $\mathrm{CV}^{2}>1$ rule does not apply to insect-pathogen interactions. A basic feature of the original models is that they do not explicitly consider the mechanisms underlying heterogeneity in attack rates (May 1978). An important conclusion of our work is thus that a consideration of such mechanisms can lead to a better understanding of species interactions.

\section{Acknowledgments}

For assistance in the field, we thank N. Aberle, C. Adler, B. Bastiaans, L. "Darth" Christ, J. Daigler, E. Fuller, J. Gent, C. Haska, R. Kelly, L. Kleczek, J. Kluse, C. O'Connell, B. "the Staff Violinist" Parker, E. Stevens, and B. Westdorp. J. Firestone spent many months and traveled many miles collecting egg masses; he also oversaw the field experiments from 2001 to 2003 and expertly summarized the raw data. M. Duffy, S. Hall, and J. Kniskern provided useful comments on the manuscript. Two anonymous reviewers also greatly improved the manuscript. Our research was supported by grants DEB-0075461 and DEB-0516327 from the National Science Foundation.

\section{Literature Cited}

Abbott, K. C., and G. Dwyer. 2007. Food limitation and insect outbreaks: complex dynamics in plant-herbivore models. Journal of Animal Ecology 76:1004-1014.

Anderson, R. M., and R. M. May. 1980. Infectious diseases and population cycles of forest insects. Science 210:658-661.

Box, G. 1979. Robustness in the strategy of scientific model building. Pages 202-236 in R. L. Launer and G. N. Wilkinson, eds. Robustness in statistics. Academic Press, New York.

Bull, J. J., R. C. Vogt, and M. G. Bulmer. 1982. Heritability of sex ratio in turtles with environmental sex determination. Evolution 36:333-341.

Capinera, J. L., K. P. Kirouac, and P. Barbosa. 1976. Phagodeterrency of cadaver components to gypsy moth larvae, Lymantria dispar. Journal of Invertebrate Pathology 28:277-279.

Cory, J. S., and J. H. Myers. 2003. The ecology and evolution of insect baculoviruses. Annual Review of Ecology and Systematics 34:239-272.

D’Amico, V., J. Elkinton, G. Dwyer, R. Willis, and M. E. Montgomery. 1998. Foliage damage does not affect within-season transmission of an insect virus. Ecology 79:1104-1110.

Duffy, M. A., and L. Sivars-Becker. 2007. Rapid evolution and ecological host-parasite dynamics. Ecology Letters 10:44-53.

Dwyer, G., and J. S. Elkinton. 1993. Using simple models to predict virus epizootics in gypsy moth populations. Journal of Animal Ecology 62:1-11.

- 1995. Host dispersal and the spatial spread of insect pathogens. Ecology 76:1262-1275.

Dwyer, G., J. S. Elkinton, and J. P. Buonaccorsi. 1997. Host heterogeneity in susceptibility and disease dynamics: tests of a mathematical model. American Naturalist 150:685-707.

Dwyer, G., J. Dushoff, J. S. Elkinton, and S. A. Levin. 2000. Pathogendriven outbreaks in forest defoliators revisited: building models from experimental data. American Naturalist 156:105-120.

Dwyer, G., J. Dushoff, J. S. Elkinton, J. P. Burand, and S. A. Levin. 2002. Variation in susceptibility: lessons from an insect virus. Pages 74-84 in U. Dieckmann, J. A. J. Metz, M. W. Sabelis, and K. Sigmund, eds. Adaptive dynamics of infectious diseases: in pursuit of virulence management. Cambridge University Press, Cambridge.

Dwyer, G., J. Dushoff, and S. H. Yee. 2004. The combined effects of pathogens and predators on insect outbreaks. Nature 430:341-345.

Dwyer, G., J. Firestone, and T. E. Stevens. 2005. Should models of disease dynamics in herbivorous insects include the effects of variability in host-plant foliage quality? American Naturalist 165:16-31.

Elkinton, J. S., and A. M. Liebhold. 1990. Population dynamics of gypsy moth in North America. Annual Review of Entomology 35: 571-596.

Erelli, M. C., and J. S. Elkinton. 2000. Maternal effects on gypsy moth (Lepidoptera: Lymantriidae) population dynamics: a field experiment. Environmental Entomology 29:476-488.

Fuxa, J. R., and A. R. Richter. 1998. Repeated reversion of resistance to nucleopolyhedrovirus by Anticarsia gemmatalis. Journal of Invertebrate Pathology 72:159-164.

Fuxa, J. R., and Y. Tanada, eds. 1987. Epizootiology of insect diseases. Wiley, New York.

Gillespie, J. H. 1975. Natural selection for resistance to epidemics. Ecology 56:493-495.

Ginzburg, L. R., and D. E. Taneyhill. 1994. Population cycles of forest 
Lepidoptera: a maternal effect hypothesis. Journal of Animal Ecology 63:79-92.

Grove, M. J., and K. Hoover. 2007. Intrastadial developmental resistance of third instar gypsy moths (Lymantria dispar L.) to $L$. dispar nucleopolyhedrovirus. Biological Control 40:355-361.

Hajek, A. E. 1999. Pathology and epizootiology of Entomophaga maimaiga infections in forest Lepidoptera. Microbiology and Molecular Biology Reviews 63:814-835.

Hassell, M. P., R. M. May, S. W. Pacala, and P. L. Chesson. 1991. The persistence of host-parasitoid associations in patchy environments. 1. A general criterion. American Naturalist 138:568-583.

Horton, H. M., and J. P. Burand. 1993. Saturable attachment sites for polyhedron-derived baculovirus on insect cells and evidence for entry via direct membrane fusion. Journal of Virology 67:1860 1868.

Hunter, A. F. 1991. Traits that distinguish outbreaking and nonoutbreaking macrolepidoptera feeding on northern hardwood trees. Oikos 60:275-282.

- 1995. Ecology, life history and phylogeny of outbreak and nonoutbreak species. Pages 41-64 in N. Cappuccino and P. Price, eds. Population dynamics: new approaches and synthesis. Academic Press, New York.

Hunter, M. D., and J. C. Schultz. 1993. Induced plant defenses breached? phytochemical induction protects an herbivore from disease. Oecologia (Berlin) 94:195-203.

Hunter-Fujita, F. R., P. F. Entwistle, H. F. Evans, and N. E. Crook. 1998. Insect viruses and pest management. Wiley, Somerset, NJ.

Johnson, D. M., A. M. Liebhold, and O. N. Bjørnstad. 2005. Circumpolar variation in periodicity and synchrony among gypsy moth populations. Journal of Animal Ecology 74:882-892.

Kermack, W., and A. McKendrick. 1927. A contribution to the mathematical theory of epidemics. Proceedings of the Royal Society A: Mathematical, Physical, and Engineering Sciences 115:700-721.

Kukan, B., and J. H. Myers. 1999. Dynamics of viral disease and population fluctuations in western tent caterpillars (Lepidoptera: Lasiocampidae) in southwestern British Columbia, Canada. Environmental Entomology 28:44-52.

Lee, K. P., J. S. Cory, K. Wilson, D. Raubenheimer, and S. J. Simpson. 2006. Flexible diet choice offsets protein costs of pathogen resistance in a caterpillar. Proceedings of the Royal Society B: Biological Sciences 273:823-829.

Liebhold, A., J. Elkinton, D. Williams, and R. M. Muzika. 2000. What causes outbreaks of the gypsy moth in North America? Population Ecology 42:257-266.

May, R. M. 1978. Host-parasitoid systems in patchy environments: a phenomenological model. Journal of Animal Ecology 47:833-844.

Mealor, M. A., and M. Boots. 2006. An indirect approach to imply trade-off shapes: population-level patterns in resistance suggest a decreasingly costly resistance mechanism in a model insect system. European Society for Evolutionary Biology 19:326-330.

Miller, L. K. 1997. The baculoviruses. Plenum, New York.

Moreau, G., and C. J. Lucarotti. 2007. A brief review of the past use of baculoviruses for the management of eruptive forest defoliators and recent developments on a sawfly virus in Canada. Forestry Chronicle 83:105-112.

Murray, K. D., and J. S. Elkinton. 1989. Environmental contamination of egg masses as a major component of transgenerational transmission of gypsy moth nuclear polyhedrosis virus (LdMNPV). Journal of Invertebrate Pathology 53:324-334.

1990. Transmission of nuclear polyhedrosis virus to gypsy moth (Lepidoptera, Lymantriidae) eggs via contaminated substrates. Environmental Entomology 19:662-665.

Myers, J. H. 1993. Population outbreaks in forest Lepidoptera. American Scientist 81:240-251.

Myers, J. H., G. H. Boettner, and J. S. Elkinton. 1998. Maternal effects in gypsy moth: only sex ratio varies with population density. Ecology 79:305-314.

Myers, J. H., R. Malakar, and J. S. Cory. 2000. Sublethal nucleopolyhedrovirus infection effects on female pupal weight, egg mass size, and vertical transmission in gypsy moth (Lepidoptera: Lymantriidae). Environmental Entomology 29:1268-1272.

Otvos, I. S., J. C. Cunningham, and L. M. Friskie. 1987. Aerial application of nuclear polyhedrosis virus against Douglas fir tussock moth, Orgyia pseudotsugata (McDunnough) (Lepidoptera: Lymantriidae). 1. Impact in the year of application. Canadian Entomologist 119:697-706.

Pawitan, Y. 2001. In all likelihood: statistical modelling and inference using likelihood. Oxford University Press, New York.

Peltonen, M., A. M. Liebhold, O. N. Bjørnstad, and D. W. Williams. 2002. Spatial synchrony in forest insect outbreaks: roles of regional stochasticity and dispersal. Ecology 83:3120-3129.

Rossiter, M. C. 1991a. Environmentally based maternal effects: a hidden force in insect population dynamics. Oecologia (Berlin) 87:288-294.

- 1991b. Maternal effects generate variation in life history: consequences of egg weight plasticity in the gypsy moth. Functional Ecology 5:386-393.

Saccheri, I., and I. Hanski. 2006. Natural selection and population dynamics. Trends in Ecology \& Evolution 21:341-347.

Schultz, J., and I. T. Baldwin. 1982. Oak leaf quality declines in response to defoliation by gypsy moth larvae. Science 217:149150 .

Shepherd, R. F., I. S. Otvos, R. J. Chorney, and J. C. Cunningham. 1984. Pest management of Douglas fir tussock moth (Lepidoptera: Lymantriidae): prevention of an outbreak through early treatment with a nuclear polyhedrosis virus by ground and aerial applications. Canadian Entomologist 116:1533-1542.

Thrall, P. H., and J. Antonovics. 1995. Theoretical and empirical studies of metapopulations: population and genetic dynamics of the Silene-Ustilago system. Canadian Journal of Botany 73:S1249S1258.

Venables, W. N., D. Smith, and the R Development Core Team. 2005. An introduction to R. Network Theory, Bristol.

Watanabe, H. 1987. The host population. Pages 71-112 in J. R. Fuxa and Y. Tanada, eds. Epizootiology of insect diseases. Wiley, New York.

Williams, D. W., and A. M. Liebhold. 1995. Influence of weather on the synchrony of gypsy moth (Lepidoptera: Lymantriidae) outbreaks in New England. Environmental Entomology 24:987-995.

Woods, S. A., and J. S. Elkinton. 1987. Bimodal patterns of mortality from nuclear polyhedrosis virus in gypsy moth (Lymantria dispar) populations. Journal of Invertebrate Pathology 50:151-157.

Associate Editor: Pejman Rohani Editor: Monica A. Geber 